(range 29-65 pg/ml). Benserazide $200 \mathrm{mg}$ orally did not reduce mean peak plasma melatonin levels or alter urinary aMT6s excretion. Peak levels and rime of peak level were similar to values in normal, healthy young laboratory staff. Mean peak plasma melatonin levels and mean urinary aMT6s excretion were slightly lower in levodopabenserazide treated than in untreated patients with Parkinson's disease ( $p>0 \cdot 1$ ). Parkinsonian disability scores (King's College rating scale), presence or absence of diurnal fatigue, peak plasma melatonin and urinary aMT6s concentrations showed no significant correlation.

The pineal gland from three patients (91 and 70 year old females, 78 year old male) with Lewy body associated Parkinson's disease, treated with levodopa-decarboxylase inhibitor combinations, were examined microscopically. Pineal histopathology was unremarkable, with cells containing brown secretory granules; there were no intracytoplasmic Lewy bodies.

Plasma melatonin and urinary 6-hydroxy melatonin sulphate concentrations in untreated and levodopa-decarboxylase inhibitor treated subjects with Parkinson's disease are within the normal range as determined in normal healthy young subjects, and it is worth noting that the pineal structure is normal in Parkinsonian patients. Therefore benserazide inhibits melatonin synthesis in the rodent but not in humans with the conventional doses used in the treatment of Parkinson's disease.

PHS CRITCHLEY GP MALCOLM PN MALCOLM WR GIBB

J ARENDT *
JD PARKES

University Department of Neurology King's College School of Medicine and Dentistry,
and Institute of Psychiatry, London Department of Biochemistry, (Division of Clinical Biochemistry)

University of Guildford, Surrey.

Correspondence to: Dr GP Malcolm, c/o Professor Symon, Institute of Neurology, National Hospital for Nervous Diseases, Queen Square, London

1 Marsden CD, Parkes JD. “On and Off”, variability and response swings in Parkinson's disease. In: Clifford-Rose F, Capildeo R, eds. Research progress in Parkinson's disease. (Progress in Neurology series). London: Pitman Medical, 1981:265-74.

2 Arendt J, Bojkowski C, Folkard S, et al. Some effects of melatonin and the control of it secretion in humans. In: Photoperiodism, melatonin, and the pineal (Ciba Foundation Symposium). London: Pitman, 1985: vol 117 266-83.

3 Arendt J, Ho AK, Laud C, et al. Differentia effect of benserazide (Ro4-4602) on the concentration on indoleamines in rat pineal and hypothalamus. Br JPharmac 1981;72:157-62.

4 Fraser S, Cowen P, Franklin M, Franey C, Arendt J. Direct radioimmunoassay for melatonin in plasma. Clin Chem 1983;29:

5 Arendt J, Bojkowski C, Franey C, Wright J Marks V. Immunoassay of 6-hydroxy melatonin sulphate in human plasma and urine: Abolition of the urinary 24-hour 1985;60:1166-73.

Cerebral CT lesions in multiple sclerosis mimicking multiple metastases

A 38 year old right handed woman was admitted with blurred vision of the right eye and incoordination of the right limbs. Eight-

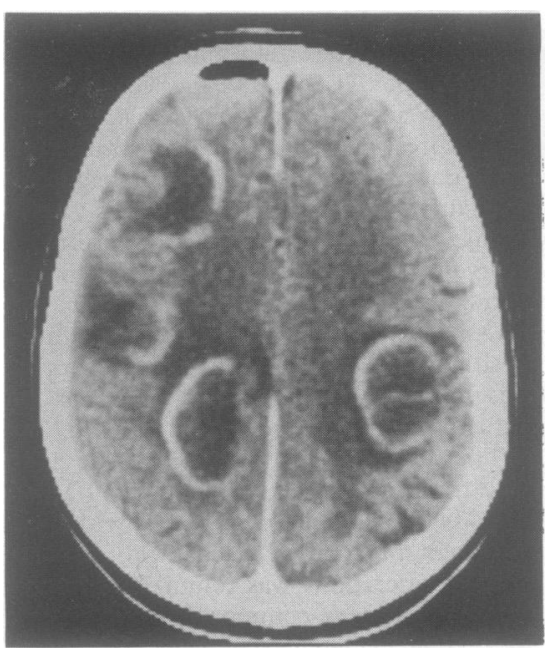

Figure Contrast enhanced CT scan showing multiple ring-like enhancing lesions.

een months previously she had developed paraesthesia and numbness of the right face and arm, with incomplete recovery and four months previously blurring of vision of the left eye with complete recovery.

Abnormal findings were an ataxic gait, VAR $6 / 18$, bilateral optic disc pallor and ataxia of the right limbs with an extensor right plantar response. Visual evoked responses showed considerably prolonged latencies from each eye. Cranial CT showed a small left frontal enhancing lesion. She was treated with high dose intravenous methylprednisolone with marked improvement.

She was re-admitted ten months later with a five month history of increasing ataxia, right hemiparesis and expressive speech difficulty. Examination showed expressive dysphasia and dysarthria. There was moderate impairment of cognitive function. Visual acuities were $6 / 60$. There was a bilateral internuclear ophthalmoplegia and right facial weakness. The right hemiparesis was severe and joint position sense was absent in the right foot. Cranial CT showed multiple ring enhancing lesions (fig). The lumbar cerebrospinal fluid (CSF) pressure was $33 \mathrm{cms}$ of water and the CSF protein was elevated at $64 \mathrm{mg} / 100 \mathrm{ml}$ with equivocal results on immunofixation. CSF contained 5 WBC per $\mathrm{mm}^{3}$, mostly lymphocytes. An HIV antibody test was negative.

There was rapid deterioration with drowsiness, dysphagia and weakness of the left arm. Because of doubt about the radiological diagnosis, and the rarity of dysphasia in multiple sclerosis, a CT guided biopsy of the left frontal lobe was carried out. The histology showed demyelination and no evidence of malignancy. She was treated with high dose intravenous methylprednisolone for five days and cyclosporin $10 \mathrm{mg} / \mathrm{kg}$ for five months with marked improvement. Six months later speech and limb power were normal and she was able to stand and walk a few steps with assistance. The CT appearances improved.

Enhancing lesions with mass effect have previously been reported in multiple sclerosis. ${ }^{1-3} \mathrm{We}$ are aware of only two reports of multiple lesions of this type ${ }^{24}$ and the florid radiological appearance in our case seems exceptional. The radiological differential diagnoses include abscesses, tumours and vascular lesions. The short term clinical and radiological improvement with corticosteroids is nonspecific and there may continue to be a need to recourse to biopsy in cases of this type.

BRF LECKY
Department of Neurology
N JEYAGOPAL
ETS SMITH
Pepartment of Neuroradiology
PM FOY
Department of Neurosurgery
Walton Hospital, Liverpool

Correspondence to: Dr Lecky, Regional Department of Neurology, Walton Hospital, Liverpoo L9 1AE, UK.

1 Van der Velden M, Bots GTAM, Endtz LJ Cranial CT in multiple sclerosis showing mass effect. Surg Neurol 1979;12:307-10.

2 Sagar HJ, Warlow CP, Sheldon PWE, Esir MM. Multiple sclerosis with clinical and radiological features of cerebral tumour. JNeurol Neurosurg Psychiatry 1982;45:802-8.

Gutling $E$, Landis T. CT ring sign imitating tumour, disclosed as multiple sclerosis by tumour, disclosed as multiple sclerosis by MRI: A Case Report.

4 Psychiatry 1989;52:903-6. $\mathrm{H}$, et al. A case of multiple sclerosis with multi ring-like and multiple sclerosis with multi ring-like and
butterfly-like enhancement on computerised butterfly-like enhancement on comp
tomography. Brain Dev 1955;7:43-5.

\section{Atypical meningitis complicating a penetrating head injury}

The bacterial genus bacillus contains predominantly saprophytic organisms with low pathogenicity for humans with the exception of the anthrax bacillus. These organisms may rarely be responsible for serious local and disseminated infections with a significant mortality. To our knowledge there have been only 11 documented cases in which bacillus subtilis has been implicated in such infections. We present a previously unrecorded complication of a penetrating head injury in which a patient survived a self-inflicted crossbow bolt injury but developed a meningitis secondary to the introduction of bacillus subtilis spores from the bolt itself.

A 24 year old man with a long history of depressive illness and drug abuse was admitted having been found in his bathroom with a crossbow by his side and a crossbow bolt protruding from his left temporal region. On examination he was conscious and orientated with no focal neurological signs and the flight feathers of the bolt were visible in the left temporal region. Skull radiographs showed the metal tip of the bolt lying in the midline (fig 1a).

The bolt was removed by a left temporal craniectomy with a perioperative dose of a broad spectrum antibiotic. The entry point was anterior to the left middle meningeal artery and the tract was fully explored and irrigated. The immediate post-operative period was uneventful. On the fifth postoperative day the patient developed a pyrexia but with no evidence of meningism. Lumbar puncture showed no evidence of organisms, a polymorph count of $180 / \mathrm{ml}$, a lymphocyte count of $10 / \mathrm{ml}$, a red cell count of $5000 / \mathrm{ml}$, a protein level of $4700 \mathrm{mg} / \mathrm{l}$ with a marked globulin increase, and a cerebrospinal fluid (CSF) glucose of $3.8 \mathrm{mmol} / 1$. Computerised tomography (CT) scan showed the bolt tract extending to the frontal horn of the left lateral 